\title{
A New Class of Meromorphically Analytic Functions with Applications to the Generalized Hypergeometric Functions
}

\author{
Firas Ghanim ${ }^{1}$ and Maslina Darus ${ }^{2}$ \\ ${ }^{1}$ Faculty of Management, Multimedia University, Cyberjaya, 63100 Selangor, Malaysia \\ 2 School of Mathematical Sciences, Faculty of Science and Technology, Universiti Kebangsaan Malaysia, \\ Bangi, 43600 Selangor, Malaysia \\ Correspondence should be addressed to Maslina Darus, maslina@ukm.my \\ Received 27 March 2011; Accepted 7 July 2011 \\ Academic Editor: Yoshikazu Giga
}

Copyright (c) 2011 F. Ghanim and M. Darus. This is an open access article distributed under the Creative Commons Attribution License, which permits unrestricted use, distribution, and reproduction in any medium, provided the original work is properly cited.

\begin{abstract}
We introduce a new subclass of meromorphically analytic functions, which is defined by means of a Hadamard product (or convolution). A characterization property such as the coefficient bound is obtained for this class. The other related properties, which are investigated in this paper, include the distortion and the radius of starlikeness. We also consider several applications of our main results to the generalized hypergeometric functions.
\end{abstract}

\section{Introduction}

Let $\mathcal{A}$ be the class of functions $f$ which are analytic in the open unit disk

$$
U=\{z \in \mathbb{C}:|z|<1\} .
$$

As usual, we denote by $S$ the subclass of $\mathcal{A}$, consisting of functions which are also univalent in $U$.

Let $w$ be a fixed point in $U$ and $A(w)=\left\{f \in H(D): f(w)=f^{\prime}(w)-1=0\right\}$. In [1], Kanas and Ronning introduced the following classes 


$$
\begin{gathered}
S_{w}=\{f \in A(w): f \text { is univalent in } U\}, \\
S T_{w}=\left\{f \in A(w): \operatorname{Re}\left(\frac{(z-w) f^{\prime}(z)}{f(z)}\right)>0, z \in U\right\}, \\
C V_{w}=\left\{f \in A: 1+\left(\operatorname{Re} \frac{(z-w) f^{\prime \prime}(z)}{f^{\prime}(z)}\right)>0, z \in U\right\} .
\end{gathered}
$$

Later, Acu and Owa [2] studied the classes extensively.

The class $S T_{w}$ is defined by geometric property that the image of any circular arc centered at $w$ is starlike with respect to $f(w)$, and the corresponding class $S_{w}^{c}$ is defined by the property that the image of any circular arc centered at $w$ is convex. We observed that the definitions are somewhat similar to the ones introduced by Goodman in $[3,4]$ for uniformly starlike and convex functions except that, in this case, the point $w$ is fixed.

Let $\Sigma_{w}$ denote the subclass of $A(w)$ consisting of the function of the form

$$
f(z)=\frac{1}{z-w}+\sum_{n=1}^{\infty} a_{n}(z-w)^{n}
$$

The functions $f$ in $\Sigma_{w}$ are said to be starlike functions of order $\beta$ if and only if

$$
\operatorname{Re}\left\{-\frac{(z-w) f^{\prime}(z)}{f(z)}\right\}>\beta \quad((z-w) \in U)
$$

for some $\beta(0 \leq \beta<1)$. We denote by $S_{w}^{*}(\beta)$ the class of all starlike functions of order $\beta$. Similarly, a function $f$ in $S_{w}$ is said to be convex of order $\beta$ if and only if

$$
\operatorname{Re}\left(-1-\frac{(z-w) f^{\prime \prime}(z)}{f^{\prime}(z)}\right)>\beta \quad((z-w) \in U)
$$

for some $\beta(0 \leq \beta<1)$. We denote by $C_{w}(\beta)$ the class of all convex functions of order $\beta$.

For the function $f \in \Sigma_{w}$, we define

$$
\begin{gathered}
I_{\lambda}^{0} f(z)=f(z), \\
I_{\lambda}^{1} f(z)=(z-w) f^{\prime}(z)+\frac{2}{z-w^{\prime}} \\
I_{\lambda}^{2} f(z)=(z-w)\left(I^{1} f(z)\right)^{\prime}+\frac{2}{z-w^{\prime}},
\end{gathered}
$$


and, for $k=1,2,3, \ldots$, we can write

$$
\begin{aligned}
I_{\lambda}^{k} f(z) & =(z-w)\left(I^{k-1} f(z)\right)^{\prime}+\frac{2}{z-w} \\
& =\frac{1}{z-w}+\sum_{n=1}^{\infty}[1+\lambda(n-1)]^{k} a_{n}(z-w)^{n},
\end{aligned}
$$

where $\lambda \geq 1, k \geq 0$ and $(z-w \in U)$.

The differential operator $I_{1}^{k}$ is studied extensively by Ghanim and Darus $[5,6]$ and Ghanim et al. [7].

The Hadamard product or convolution of the functions $f$ given by (1.3) with the function $g$ and $h$ given, respectively, by

$$
\begin{aligned}
& g(z)=\frac{1}{z-w}+\sum_{n=1}^{\infty} b_{n}(z-w)^{n}, \\
& h(z)=\frac{1}{z-w}+\sum_{n=1}^{\infty} c_{n}(z-w)^{n},
\end{aligned}
$$

can be expressed as follows:

$$
\begin{aligned}
& (f * g)(z)=\frac{1}{z-w}+\sum_{n=1}^{\infty} a_{n} b_{n}(z-w)^{n}, \\
& (f * h)(z)=\frac{1}{z-w}+\sum_{n=1}^{\infty} a_{n} c_{n}(z-w)^{n} .
\end{aligned}
$$

Suppose that $f$ and $g$ are two analytic functions in the unit disk $U$. Then, we say that the function $g$ is subordinate to the function $f$, and we write

$$
g(z) \prec f(z) \quad(z \in U)
$$

if there exists a Schwarz function $\varpi(z)$ with $\varpi(0)=0$ and $|\varpi(z)|<1$ such that

$$
g(z)=f(\varpi(z)) \quad(z \in U)
$$

By applying the above subordination definition, we introduce here a new class $\Sigma_{w}(A$, $B, k, \alpha, \lambda)$ of meromorphically functions, which is defined as follows:

Definition 1.1. A function $f \in \Sigma_{w}$ of the form (1.3) is said to be in the class $\Sigma_{w}(A, B, k, \alpha, \lambda)$ if it satisfies the following subordination property:

$$
\alpha \frac{I_{\lambda}^{k}(f * g)(z)}{I_{\lambda}^{k}(f * h)(z)} \prec \alpha-\frac{(A-B)(z-w)}{1+B(z-w)} \quad((z-w) \in U)
$$

where $-1 \leq B<A \leq 1, k \geq 0, \alpha>0, \lambda \geq 1$, with condition $I_{\lambda}^{k}(f * h)(z) \neq 0$. 
The purpose of this paper is to investigate the coefficient estimates, distortion properties, and the radius of starlikeness for the class $\Sigma_{w}(A, B, k, \alpha, \lambda)$. Some applications of the main results involving generalized hypergeometric functions are also considered.

\section{Characterization and Other Related Properties}

In this section, we begin by proving a characterization property which provides a necessary and sufficient condition for a function $f \in \Sigma_{w}$ of the form (1.3) to belong to the class $\Sigma_{w}(A, B, k, \alpha, \lambda)$ of meromorphically analytic functions.

Theorem 2.1. The function $f \in \Sigma_{w}$ is said to be a member of the class $\Sigma_{w}(A, B, k, \alpha, \lambda)$ if it satisfies

$$
\sum_{n=1}^{\infty}[1+\lambda(n-1)]^{k}\left(\alpha b_{n}(1+B)-c_{n}(\alpha(1+B)+A-B)\right) a_{n} \leq A-B
$$

The equality is attained for the function $f_{n}(z)$ given by

$$
f_{n}(z)=\frac{1}{z-w}+\frac{(A-B)}{[1+\lambda(n-1)]^{k}\left(\alpha b_{n}(1+B)-c_{n}(\alpha(1+B)+A-B)\right)}(z-w)^{n} .
$$

Proof. Let $f \in \Sigma_{w}(A, B, k, \alpha, \lambda)$, and suppose that

$$
\alpha \frac{I_{\lambda}^{k}(f * g)(z)}{I_{\lambda}^{k}(f * h)(z)}=\alpha-\frac{(A-B)(z-w)}{1+B(z-w)}
$$

Then, in view of (2.2), we have

$$
\begin{aligned}
& \left|\frac{\alpha \sum_{n=1}^{\infty}[1+\lambda(n-1)]^{k} a_{n}\left(b_{n}-c_{n}\right)(z-w)^{n+1}}{(A-B)-\sum_{n=1}^{\infty}[1+\lambda(n-1)]^{k} a_{n}\left(\alpha B b_{n}+\{(A-B)-\alpha B\} c_{n}\right)(z-w)^{n+1}}\right| \\
& \quad \leq \frac{\alpha \sum_{n=1}^{\infty}[1+\lambda(n-1)]^{k} a_{n}\left(b_{n}-c_{n}\right)|z-w|^{n+1}}{(A-B)-\sum_{n=1}^{\infty}[1+\lambda(n-1)]^{k} a_{n}\left(\alpha B b_{n}+\{(A-B)-\alpha B\} c_{n}\right)|z-w|^{n+1}} \\
& \quad \leq 1 .
\end{aligned}
$$

Letting $(z-w) \rightarrow 1$, we get

$$
\sum_{n=1}^{\infty}[1+\lambda(n-1)]^{k}\left(\alpha b_{n}(1+B)-c_{n}(\alpha(1+B)+A-B)\right) a_{n} \leq(A-B)
$$

which is equivalent to our condition of the theorem, so that $f \in \Sigma_{w}(A, B, k, \alpha, \lambda)$. Hence we have the theorem. 
Theorem 2.1 immediately yields the following result.

Corollary 2.2. If the function $f \in \Sigma_{w}$ belongs to the class $\Sigma_{w}(A, B, k, \alpha, \lambda)$, then

$$
a_{n} \leq \frac{(A-B)}{[1+\lambda(n-1)]^{k}\left(\alpha b_{n}(1+B)-c_{n}(\alpha(1+B)+A-B)\right)},
$$

$n \geq 1$, where the equality holds true for the functions $f_{n}(z)$ given by (2.2).

We now state the following growth and distortion properties for the class $\Sigma_{w}(A$, $B, k, \alpha, \lambda)$.

Theorem 2.3. If the function $f$ defined by (1.3) is in the class $\Sigma_{w}(A, B, k, \alpha, \lambda)$, then for $0<|z-w|=$ $r<1$, one has

$$
\begin{aligned}
& \frac{1}{r}-\frac{(A-B)}{\left(\alpha b_{1}(1+B)-c_{1}(\alpha(1+B)+A-B)\right)} r \\
& \quad \leq|f(z)| \leq \frac{1}{r}+\frac{(A-B)}{\left(\alpha b_{1}(1+B)-c_{1}(\alpha(1+B)+A-B)\right)} r, \\
& \frac{1}{r^{2}}-\frac{(A-B)}{\left(\alpha b_{1}(1+B)-c_{1}(\alpha(1+B)+A-B)\right)} \\
& \quad \leq\left|f^{\prime}(z)\right| \leq \frac{1}{r^{2}}+\frac{(A-B)}{\left(\alpha b_{1}(1+B)-c_{1}(\alpha(1+B)+A-B)\right)} .
\end{aligned}
$$

Proof. Since $f \in \Sigma_{w}(A, B, k, \alpha, \lambda)$, Theorem 2.1 readily yields the inequality

$$
\sum_{n=1}^{\infty} a_{n} \leq \frac{(A-B)}{\left(\alpha b_{1}(1+B)-c_{1}(\alpha(1+B)+A-B)\right)} .
$$

Thus, for $0<|z-w|=r<1$ and utilizing (2.8), we have 


$$
\begin{aligned}
|f(z)| & \leq \frac{1}{|z-w|}+\sum_{n=1}^{m} a_{n}|(z-w)|^{n} \\
& \leq \frac{1}{r}+r \sum_{n=1}^{m} a_{n} \\
& \leq \frac{1}{r}+\frac{(A-B)}{\left(\alpha b_{1}(1+B)-c_{1}(\alpha(1+B)+A-B)\right)} r, \\
|f(z)| & \geq \frac{1}{|z-w|}-\sum_{n=1}^{m} a_{n}|(z-w)|^{n} \\
& \geq \frac{1}{r}-r \sum_{n=1}^{m} a_{n} \quad(A-B) \\
& \geq \frac{1}{r}-\frac{\left(\alpha b_{1}(1+B)-c_{1}(\alpha(1+B)+A-B)\right)}{(1+.} .
\end{aligned}
$$

Also, from Theorem 2.1, we get

$$
\sum_{n=1}^{\infty} n a_{n} \leq \frac{(A-B)}{\left(\alpha b_{1}(1+B)-c_{1}(\alpha(1+B)+A-B)\right)} .
$$

Hence

$$
\begin{aligned}
\left|f^{\prime}(z)\right| & \leq \frac{1}{|z-w|^{2}}+\sum_{n=1}^{m} n a_{n}|(z-w)|^{n-1} \\
& \leq \frac{1}{r}+\sum_{n=1}^{m} n a_{n} \\
& \leq \frac{1}{r^{2}}+\frac{(A-B)}{\left(\alpha b_{1}(1+B)-c_{1}(\alpha(1+B)+A-B)\right)}, \\
\left|f^{\prime}(z)\right| & \geq \frac{1}{|z-w|^{2}}-\sum_{n=1}^{m} n a_{n}|(z-w)|^{n-1} \\
& \geq \frac{1}{r^{2}}-\sum_{n=1}^{m} n a_{n} \quad(A-B) \\
& \geq \frac{1}{r^{2}}-\frac{\left(\alpha b_{1}(1+B)-c_{1}(\alpha(1+B)+A-B)\right)}{}
\end{aligned}
$$

This completes the proof of Theorem 2.3. 
We next determine the radius of meromorphically starlikeness of the class $\Sigma_{w}(A$, $B, k, \alpha, \lambda)$, which is given by Theorem 2.4 .

Theorem 2.4. If the function $f$ defined by (1.3) is in the class $\Sigma_{w}(A, B, k, \alpha, \lambda)$, then $f$ is meromorphically starlike of order $\delta$ in the disk $|z-w|<r_{1}$, where

$$
r_{1}=\inf _{n \geq 1}\left\{\frac{(1-\delta)\left(\alpha b_{n}(1+B)-c_{n}(\alpha(1+B)+A-B)\right)}{(n+2-\delta)(A-B)}\right\}^{1 /(n+1)}
$$

The equality is attained for the function $f_{n}(z)$ given by (2.2).

Proof. It suffices to prove that

$$
\left|\frac{(z-w)\left(I^{k} f(z)\right)^{\prime}}{I^{k} f(z)}+1\right| \leq 1-\delta
$$

For $|z-w|<r_{1}$, we have

$$
\begin{aligned}
\left|\frac{(z-w)\left(I^{k} f(z)\right)^{\prime}}{I^{k} f(z)}+1\right| & =\left|\frac{\sum_{n=1}^{\infty}(n+1)[1+\lambda(n-1)]^{k} a_{n}(z-w)^{n}}{1 /(z-w)+\sum_{n=1}^{\infty}[1+\lambda(n-1)]^{k} a_{n}(z-w)^{n}}\right| \\
& =\left|\frac{\sum_{n=1}^{\infty}(n+1)[1+\lambda(n-1)]^{k} a_{n}(z-w)^{n+1}}{1+\sum_{n=1}^{\infty}[1+\lambda(n-1)]^{k} a_{n}(z-w)^{n+1}}\right| \\
& \leq \frac{\sum_{n=1}^{\infty}(n+1)[1+\lambda(n-1)]^{k} a_{n}|z-w|^{n+1}}{1-\sum_{n=1}^{\infty}[1+\lambda(n-1)]^{k} a_{n}|z-w|^{n+1}} .
\end{aligned}
$$

Hence (2.14) holds true for

$$
\sum_{n=1}^{\infty}(n+1)[1+\lambda(n-1)]^{k} a_{n}|z-w|^{n+1} \leq(1-\delta)\left(1-\sum_{n=1}^{\infty}[1+\lambda(n-1)]^{k} a_{n}|z-w|^{n+1}\right)
$$

or

$$
\frac{\sum_{n=1}^{\infty}(n+2-\delta)[1+\lambda(n-1)]^{k} a_{n}|z-w|^{n+1}}{(1-\delta)} \leq 1
$$

With the aid of (2.1) and (2.16), it is true to say that for fixed $n$

$$
\begin{aligned}
& \frac{(n+2-\delta)[1+\lambda(n-1)]^{k}|z-w|^{n+1}}{(1-\delta)} \\
& \quad \leq \frac{[1+\lambda(n-1)]^{k}\left(\alpha b_{n}(1+B)-c_{n}(\alpha(1+B)+A-B)\right)}{(A-B)} \quad(n \geq 1) .
\end{aligned}
$$


Solving (2.17) for $|z-w|$, we obtain

$$
|z-w|<\left\{\frac{(1-\delta)\left(\alpha b_{n}(1+B)-c_{n}(\alpha(1+B)+A-B)\right)}{(n+2-\delta)(A-B)}\right\}^{1 /(n+1)}
$$

This completes the proof of Theorem 2.4.

\section{Applications Involving Generalized Hypergeometric Functions}

Let us define the function $\tilde{\phi}(a, c ; z)$ by

$$
\tilde{\phi}(a, c ; z)=\frac{1}{z-w}+\sum_{n=0}^{\infty}\left|\frac{(a)_{n+1}}{(c)_{n+1}}\right| a_{n}(z-w)^{n}
$$

for $c \neq 0,-1,-2, \ldots$, and $a \in \mathbb{C} /\{0\}$, where $(\lambda) n=\lambda(\lambda+1)_{n+1}$ is the Pochhammer symbol. We note that

$$
\tilde{\phi}(a, c ; z)=\frac{1}{z-w}{ }_{2} F_{1}(1, a, c ; z)
$$

where

$$
{ }_{2} F_{1}(b, a, c ; z)=\sum_{n=0}^{\infty} \frac{(b)_{n}(a)_{n}}{(c)_{n}} \frac{(z-w)^{n}}{n !} .
$$

Corresponding to the function $\tilde{\phi}(a, c ; z)$ and using the Hadamard product which was defined earlier in the introduction section for $f(z) \in \Sigma$, we define here a new linear operator $L^{*}(a, c)$ on $\Sigma$ by

$$
L_{w}^{*}(a, c) f(z)=\tilde{\phi}(a, c ; z) * f(z)=\frac{1}{z-w}+\sum_{n=1}^{\infty}\left|\frac{(a)_{n+1}}{(c)_{n+1}}\right| a_{n}(z-w)^{n}
$$

For a function $f \in L_{w}^{*}(a, c) f(z)$, we define

$$
I^{0}\left(L_{w}^{*}(a, c) f(z)\right)=L_{w}^{*}(a, c) f(z)
$$

and, for $k=1,2,3, \ldots$,

$$
\begin{aligned}
I^{k}\left(L_{w}^{*}(a, c) f(z)\right) & =z\left(I^{k-1} L^{*}(a, c) f(z)\right)^{\prime}+\frac{2}{z-w} \\
& =\frac{1}{z-w}+\sum_{n=1}^{\infty} n^{k}\left|\frac{(a)_{n+1}}{(c)_{n+1}}\right| a_{n}(z-w)^{n}
\end{aligned}
$$


We note $I^{k}\left(L_{w}^{*}(a, a) f(z)\right)$ studied by Ghanim and Darus [5, 6] and Ghanim et al. [7], and also, $I^{k}\left(L_{0}^{*}(a, c) f(z)\right)$ studied by Ghanim and Darus [8,9] and Ghanim et al. [10].

The subordination relation (1.12) in conjunction with (3.4) and (3.6) takes the following form:

$$
\alpha \frac{I^{k} L_{w}^{*}(a+1, c) f(z)}{I^{k} L_{w}^{*}(a, c) f(z)}=\alpha-\frac{(A-B)(z-w)}{1+B(z-w)}
$$

$(0 \leq B<A \leq 1, k \geq 0, \alpha>0)$.

Definition 3.1. A function $f \in \Sigma_{w}$ of the form (1.3) is said to be in the class $\Sigma_{w}(A, B, k, \alpha, a, c)$ if it satisfies the subordination relation (3.7) above.

Theorem 3.2. The function $f \in \Sigma_{w}$ is said to be a member of the class $\Sigma_{w}(A, B, k, \alpha, a, c)$ if it satisfies

$$
\sum_{n=1}^{\infty} n^{k}\left(\alpha b_{n}(1+B)-c_{n}(\alpha(1+B)+A-B)\right) \frac{\left|(a)_{n+1}\right|}{\left|(c)_{n+1}\right|} a_{n} \leq(A-B) .
$$

The equality is attained for the function $f_{n}(z)$ given by

$$
f_{n}(z)=\frac{1}{z-w}+\sum_{n=1}^{\infty} \frac{(A-B)\left|(c)_{n+1}\right|}{n^{k}\left(\alpha b_{n}(1+B)-c_{n}(\alpha(1+B)+A-B)\right)\left|(a)_{n+1}\right|}(z-w)^{n}
$$

$n \geq 1$.

Proof. By using the same technique employed in the proof of Theorem 2.1 along with Definition 3.1, we can prove Theorem 3.2.

The following consequences of Theorem 3.2 can be deduced by applying (3.8) and (3.9) along with Definition 3.1.

Corollary 3.3. If the function $f \in \Sigma_{w}$ belongs to the class $\Sigma_{w}(A, B, k, \alpha, a, c)$, then

$$
a_{n} \leq \frac{(A-B)\left|(c)_{n+1}\right|}{n^{k}\left(\alpha b_{n}(1+B)-c_{n}(\alpha(1+B)+A-B)\right)\left|(a)_{n+1}\right|}
$$

$n \geq 1$, where the equality holds true for the functions $f_{n}(z)$ given by (3.9).

Corollary 3.4. If the function $f$ defined by (1.3) is in the class $\Sigma_{w}(A, B, k, \alpha, a, c)$, then $f$ is meromorphically starlike of order $\delta$ in the disk $|z-w|<r_{3}$, where

$$
r_{3}=\inf _{n \geq 1}\left\{\frac{(1-\delta)\left(\alpha b_{n}(1+B)-c_{n}(\alpha(1+B)+A-B)\right)\left|(c)_{n+1}\right|}{(n+2-\delta)(A-B)\left|(a)_{n+1}\right|}\right\}^{1 /(n+1)} .
$$

The equality is attained for the function $f_{n}(z)$ given by (3.9). 
A slight background related to the formation of the present operator can be found in [11], and other work can be tackled using this type of operator. Also, the meromorphic functions with the generalized hypergeometric functions were considered recently by Dziok and Srivastava [12, 13], Liu [14], Liu and Srivastava [15], and Cho and Kim [16].

\section{Acknowledgment}

The work presented here was fully supported by UKM-ST-06-FRGS0244-2010.

\section{References}

[1] S. Kanas and F. Ronning, "Uniformly starlike and convex functions and other related classes of univalent functions," Annales Universitatis Mariae Curie-Sklodowska, vol. 53, pp. 95-105, 1991.

[2] M. Acu and S. Owa, "On some subclasses of univalent functions," Journal of Inequalities in Pure and Applied Mathematics, vol. 6, no. 3, pp. 1-6, 2005.

[3] A. W. Goodman, "On uniformly starlike functions," Journal of Mathematical Analysis and Applications, vol. 155, no. 2, pp. 364-370, 1991.

[4] A. W. Goodman, “On uniformly convex functions," Annales Polonici Mathematici, vol. 56, no. 1, pp. 87-92, 1991.

[5] F. Ghanim and M. Darus, "On certain class of analytic function with fixed second positive coefficient," International Journal of Mathematical Analysis, vol. 2, no. 2, pp. 55-66, 2008.

[6] F. Ghanim and M. Darus, "Some subordination results associated with certain subclass of analytic meromorphic functions," Journal of Mathematics and Statistics, vol. 4, no. 2, pp. 112-116, 2008.

[7] F. Ghanim, M. Darus, and S. Sivasubramanian, "On new subclass of analytic univalent function," International Journal of Pure and Applied Mathematics, vol. 40, no. 3, pp. 307-319, 2007.

[8] F. Ghanim and M. Darus, "Linear operators associated with a subclass of hypergeometric meromorphic uniformly convex functions," Acta Universitatis Apulensis, no. 17, pp. 49-60, 2009.

[9] F. Ghanim and M. Darus, "Certain subclasses of meromorphic functions related to Cho-KwonSrivastava operator," Far East Journal of Mathematical Sciences, vol. 48, no. 2, pp. 159-173, 2011.

[10] F. Ghanim, M. Darus, and A. Swaminathan, "New subclass of hypergeometric meromorphic functions," Far East Journal of Mathematical Sciences, vol. 34, no. 2, pp. 245-256, 2009.

[11] B. A. Frasin and M. Darus, "On certain meromorphic functions with positive coefficients," Southeast Asian Bulletin of Mathematics, vol. 28, no. 4, pp. 615-623, 2004.

[12] J. Dziok and H. M. Srivastava, "Some subclasses of analytic functions with fixed argument of coefficients associated with the generalized hypergeometric function," Advanced Studies in Contemporary Mathematics, vol. 5, no. 2, pp. 115-125, 2002.

[13] J. Dziok and H. M. Srivastava, "Certain subclasses of analytic functions associated with the generalized hypergeometric function," Integral Transforms and Special Functions, vol. 14, no. 1, pp. 7-18, 2003.

[14] J. L. Liu, "A linear operator and its applications on meromorphic p-valent functions," Bulletin of the Institute of Mathematics, vol. 31, no. 1, pp. 23-32, 2003.

[15] J. L. Liu and H. M. Srivastava, "Certain properties of the Dziok-Srivastava operator," Applied Mathematics and Computation, vol. 159, no. 2, pp. 485-493, 2004.

[16] N. E. Cho and I. H. Kim, "Inclusion properties of certain classes of meromorphic functions associated with the generalized hypergeometric function," Applied Mathematics and Computation, vol. 187, no. 1, pp. 115-121, 2007. 


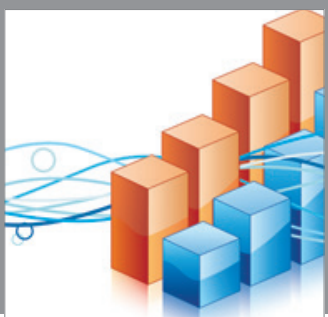

Advances in

Operations Research

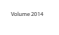

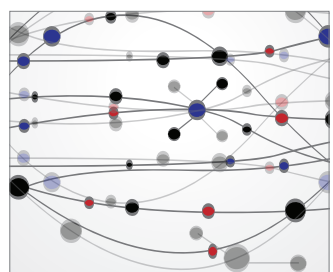

\section{The Scientific} World Journal
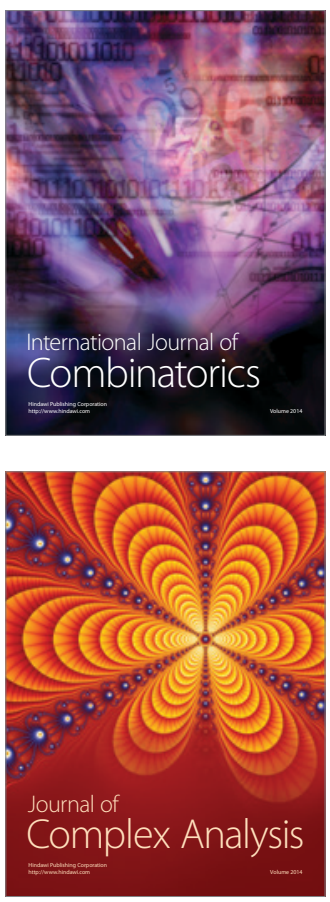

International Journal of

Mathematics and

Mathematical

Sciences
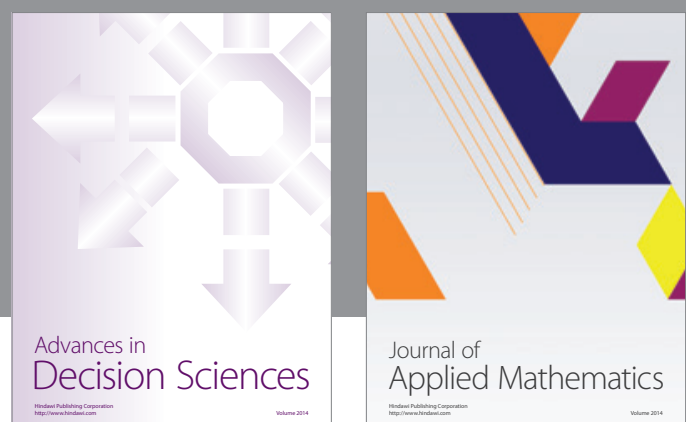

Journal of

Applied Mathematics
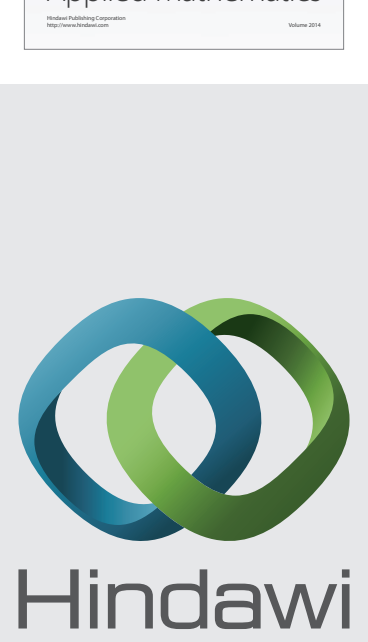

Submit your manuscripts at http://www.hindawi.com
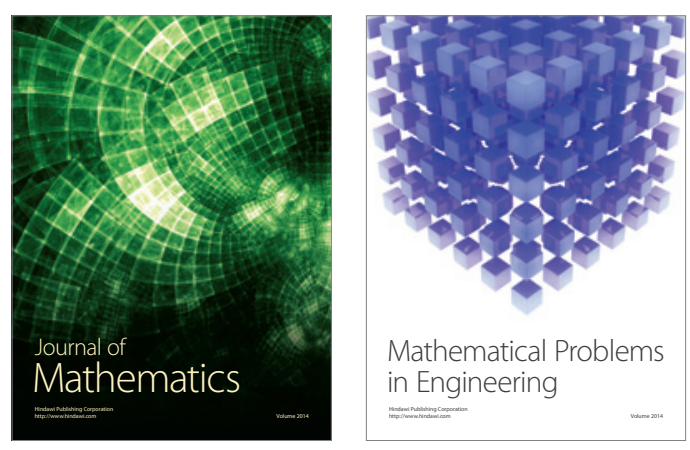

Mathematical Problems in Engineering
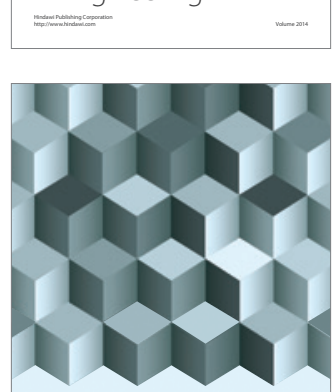

Journal of

Function Spaces
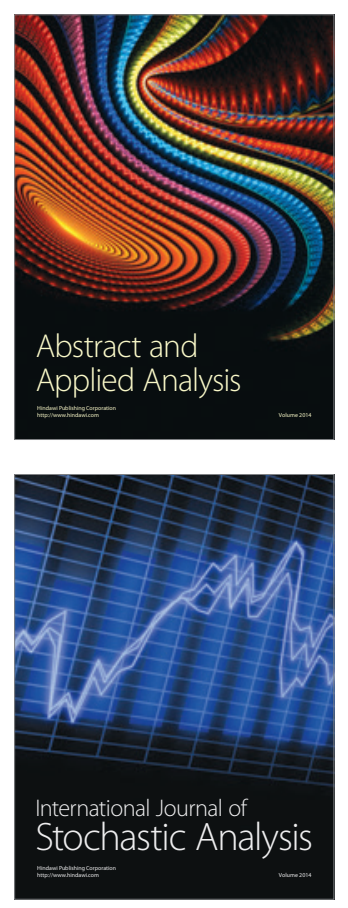

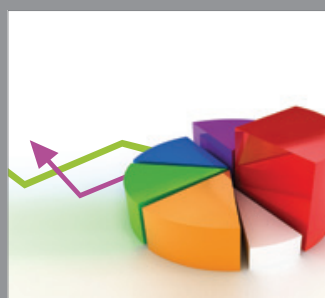

ournal of

Probability and Statistics

Promensencen
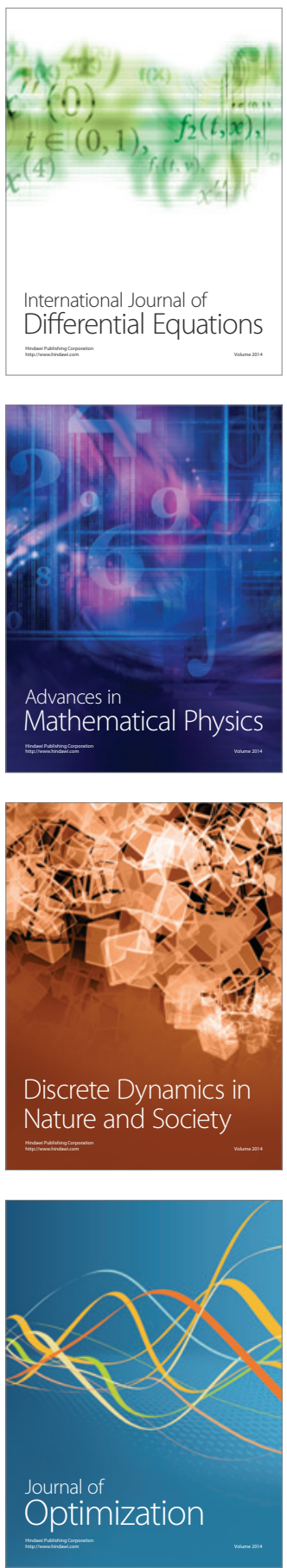